\title{
Implications of compressibility effects for Reynolds-scaled testing of an inverted wing in ground effect
}

\section{Graham Doig* and Tracie J. Barber}

School of Mechanical and Manufacturing Engineering, The University of New South Wales, NSW 2052, Sydney, Australia

Email: grahamdoig@hotmail.com

Email: t.barber@unsw.edu.au

${ }^{*}$ Corresponding author

\section{Sammy Diasinos}

Department of Engineering, Macquarie University, North Ryde, NSW 2109, Sydney, Australia Email: sammy.diasinos@mq.edu.au

Abstract: The influence of compressibility around an isolated inverted wing at a fixed Reynolds number was examined as relevant to the issue of wind tunnel scaling effects. Three-dimensional simulations were conducted for low ground clearances, at: full scale and a Mach number of 0.088 , at $50 \%$ scale at Mach 0.176 , and at $25 \%$ scale at Mach 0.352 . As the scale was reduced, the increasing peak local Mach number between the wing and the ground resulted in a higher propensity of the flow to separate towards the trailing edge, and for incompressible or full-scale CFD to underestimate the lift and drag coefficients by an ever-increasing margin. The lower vortex path was less affected. The results suggest that compressible CFD of a scale experiment ought to be conducted at the same Reynolds number and Mach number as the tunnel test for the best possible correlation at free-stream Mach numbers beyond 0.15 .

Keywords: ground effect; $\mathrm{CFD}$; compressible flow; Reynolds-scaling; inverted wing; downforce.

Reference to this paper should be made as follows: Doig, G., Barber, T.J. and Diasinos, S. (2014) 'Implications of compressibility effects for Reynolds-scaled testing of an inverted wing in ground effect', Int. J. Aerodynamics, Vol. 4, Nos. 3/4, pp.135-153.

Biographical notes: Graham Doig is a Lecturer at the University of New South Wales (UNSW), where he is also the Academic Supervisor of the Sunswift Solar Car Racing Team. He received his MEng from the University of Glasgow and his PhD from UNSW, and subsequently worked as a Mechanical Engineer for AECOM before rejoining UNSW. His current research areas include transient automotive aerodynamics and high speed flows, with a focus on synergies betwcen experimental and numerical techniques. 
Tracie J. Barber's research in ground effect flow aerodynamics uses both computational and experimental methods, with an emphasis on the integration of the two procedures. She has over 100 publications in the area. Her interests cover modelling methodologies, subsonic ground effect aerodynamics and compressible flow ground effect aerodynamics. She is also involved in work in the area of vascular fluid dynamics, covering both experimental and computational investigations. She is a Researcher at the University of New South Wales, where she is also the Director of Laboratories for the School of Mechanical and Manufacturing Engineering.

Sammy Diasinos is a Lecturer at Macquarie University who completed his MEng and PhD at University of New South Wales. Ile was then employed by the Toyota and Williams Formula One $(\mathrm{F} 1)$ teams in a variety of computational and experimental engineering roles, eventually becoming the Head of CFD Development for the Caterham F1 team. Upon returning to academia, he has been pursuing his research interests in aerodynamics of sport and the automotive industry, with an emphasis in understanding and extending the limitations of both experimental and computational approaches related to these fields.

\section{Introduction}

A designer has three options for examining the aerodynamic characteristics of a racing car or its bodywork components: wind tunnel testing with a model, CFD simulation, and track testing with an actual vehicle. The latter is rare in the context of ongoing design development (Zhang et al., 2006), and the former two are usually performed in close concert with CFD both filling in gaps in tunnel testing and simulating scenarios not possible in experimental conditions (Toet, 2013).

When it comes to racing car testing and simulation, compressibility effects have yet to be properly investigated and accounted for (Zhang et al., 2006). Given that a wing in ground effect can produce local velocities at its suction peak significantly higher than those produced out of ground effect, the Mach numbers in such flowfields may result in significant density changes in the air between the wing and ground.

There are many factors which can influence the fidelity of comparisons between wind tunnel data and CFD simulations; for instance sting interference, wall interference, and errors inherent to the measurement techniques and those which stem from tunnel calibration. In Reynolds-averaged Navier-stokes (RANS) CFD, errors arise from the mesh, turbulence model and treatment of transition, the Reynolds-averaging process itself in calculating a steady flowfield, and the numerous simplifications often made such as elimination of stings and small-scale geometric features.

In the midst of these other factors and potential sources of uncertainty, the influence of density changes in the flow could be masked even if they are as important as recent studies on a single-element airfoil and wing indicate (Doig et al., 2007, 2011). Those results showed that incompressible simulations could underpredict drag and lift forces of an inverted wing in ground effect by several percent even at a freestream Mach number of 0.15. The experiments of Ranzenbach (1995) and Ranzenbach and Barlow (1994, $1995,1996,1997)$, were conducted at a freestream velocity of approximately $45 \mathrm{~ms}^{-1}$ and compared to incompressible CFD. The Mach number in that instance was between 
0.13 and 0.14 , which the authors explicitly state was chosen so as to avoid introducing significant compressibility effects in the accelerated region, though the possible extent of such effects was not speculated upon.

As an illustration of the relevance of the study described in this paper, the Formula 1 regulations have stated since 2010 that "no wind tunnel testing may be carried out using a scale model which is greater than 60 percent of full size" and "no wind tunnel testing may be carried out at a speed exceeding 50 metres/second" (FIA, 2013). These are measures essentially designed to reduce costs. If testing is conducted with $50 \%$ scale models, the $50 \mathrm{~ms}^{-1}$ tunnel speed would equate to $25 \mathrm{~ms}^{-1}$ for a full-scale CFD model at the same Reynolds number.

The study by Doig et al. (2011) described a comparison between compressible and incompressible CFD loosely equivalent to comparing incompressible CFD to results obtained by a full-scale vehicle on track as the Reynolds number was left to vary freely with increasing velocity. The study did not consider the influence of Reynolds number as separated from that of Mach number, and therefore neglected the scenario relevant to testing development whereby Reynolds-scaled models are used and matched to full-scale (incompressible) modelling at a different Mach number. The present study outlines a similar scenario, but one in which the goal would be to correlate full-scale CFD to scale-model wind tunnel tests, and where precise Reynolds-scaling may be perceived as being sufficient for comparisons due to the relatively low speeds involved. This scenario is industry-relevant, and the parameters considered are outlined in Table 1. While flow features such as transition and separation - traditionally difficult to predict with RANS CFD - would be more influential in correlation issues, it is proposed that compressibility effects are relatively easy to account for in simulations and therefore worth quantifying.

Table 1 List of Mach and Reynolds numbers and related parameters

\begin{tabular}{lccccc}
\hline Mach no. & Velocity $\left(\mathrm{ms}^{-1}\right)$ & $\mathrm{km} / \mathrm{h}$ & $m p h$ & $R e$ & Scale \\
\hline 0.882 & 30 & 108 & 67.1 & $0.459 \times 10^{6}$ & $100 \%$ \\
0.1764 & 60 & 216 & 136.2 & $0.459 \times 10^{6}$ & $50 \%$ \\
0.3528 & 120 & 432 & 268.4 & $0.459 \times 10^{6}$ & $25 \%$ \\
\hline
\end{tabular}

If a body is slender and the flowfield is sufficiently two-dimensional as one would expect of the mid-span of a non-tapered, non-swept wing, the Prandtl-Glauert rule may be considered applicable (Glauert, 1928), and the effectiveness of this as a straightforward compressible correction is examined compared to the CFD-predictions.

\section{Methodology}

A commercially-available finite-volume RANS solver, ANSYS fluent, was used to obtain all of the data. Steady-state solutions were generated using the implicit, pressure-based, coupled solver.

Convergence criteria for all simulations were deemed to be met not only when the mass and momentum scaled-residual errors changed by less than $0.01 \%$ over 1,000 continued iterations, but also when the aerodynamic force coefficients on the body ceased to change by the same percentage over that period. Results were obtained using 64-bit double precision and a second order node-based upwinding discretisation scheme. A 
standard three-coefficient Sutherland (1893) viscosity model was applied to simulations involving compressible flow although the influence of this modelling choice was not significant at such relatively low Mach numbers.

A fully-structured hexahedral grid was generated for each individual ground clearance. The coordinates of the original wing can be found in Zerihan (2001); it was a modified LS(1)-0413 MOD section, with a constant cross section chord of $223.4 \mathrm{~mm}$ and a span of $1,100 \mathrm{~mm}(75 \%$ of the moving ground width), and featured a basic endplate $(250 \times 100 \times 4 \mathrm{~mm})$. The aspect ratio of the wing was 4.92 , representing a wing approximately $80 \%$ of a more complex, full-scale Formula 1 wing. The trailing edge of the main section was blunted to a finite $0.007 \mathrm{c}$ thickness. Some relevant characteristics of the wing in ground effect are shown in Figure 1.

Figure 1 Notation for an inverted wing (section) in ground effect

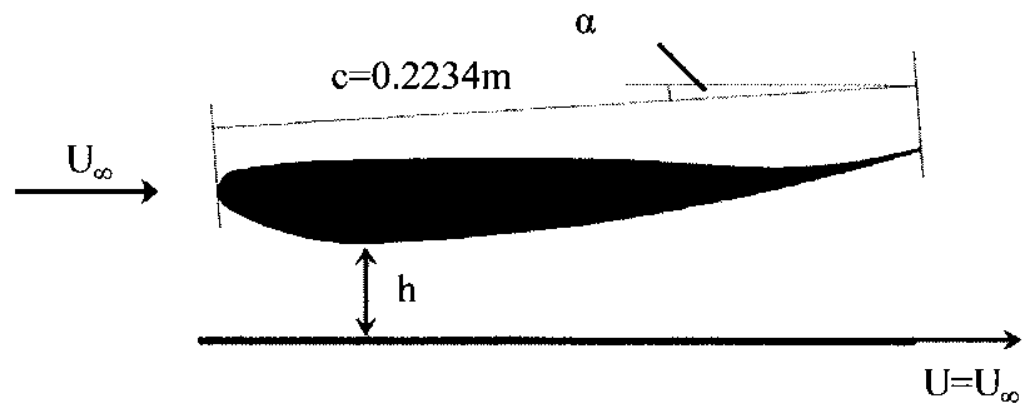

All cases were run as semi-span models with a symmetry plane in the middle of the wing $(\mathrm{z}=0)$. For the present manuscript, the coordinate origin is at the leading edge of the wing on the symmetry plane (semi-span). The boundaries of the wind tunnel $(2.1 \times 1.7 \mathrm{~m})$ were used, although with a rectangular cross-section rather than the octagon used in the actual tunnel, as this has been shown in recent literature to have negligible influence on the results (Diasinos et al., 2013).

The oncoming air was set at $30 \mathrm{~ms}^{-t}$ (as was the moving ground simulating the belt in the tunnel), and the chord-based Reynolds number for all experiments, was in the range of $0.43-0.46 \times 10^{6}$. In the present CFD, density was kept at a constant and the Reynolds number was matched for validation purposes.

The wing was held at a reference incidence of $3.45^{\circ}$ (anticlockwise, or nose-downwards rotation from horizontal), with the ground clearance, defined in terms of $\mathrm{h} / \mathrm{c}$, measured from the point on the wing surface closest to the ground plane, as shown in the schematic of Figure 1. In the original experiments, freestream turbulence intensity was measured as being $0.2 \%$, and boundary layer transition on the wing was tripped with a grit strip at $10 \%$ of the chord from the leading edge - this feature was accounted for in the present CFD by crude introduction of a laminar zone ahead of $10 \% \mathrm{c}$. For the purposes of the numerical model, the test section was assumed to extend $7 \mathrm{c}$ upstream of the leading edge, and $15 \mathrm{c}$ downstream.

Mesh nodes were concentrated in the vicinity of the wing and the endplate, with anisotropic cells growing from the wing and ground boundaries to better capture the boundary layer. The wall $y^{+}$was between 1 and 2 on the wing when flow was attached, enabling high-resolution wall modelling. Coarse, standard and fine meshes were 
evaluated against the experimental data for $\mathrm{h} / \mathrm{c}=0.179, \mathrm{U}_{\infty}=30 \mathrm{~ms}^{-1}$. Similar meshes were constructed for some other clearances but the results at $h / c=0.179$ are suitably representative of all cases. The general meshing strategy is shown in Figure 2.

Figure 2 Example of the structured mesh around the semi-span wing with ground

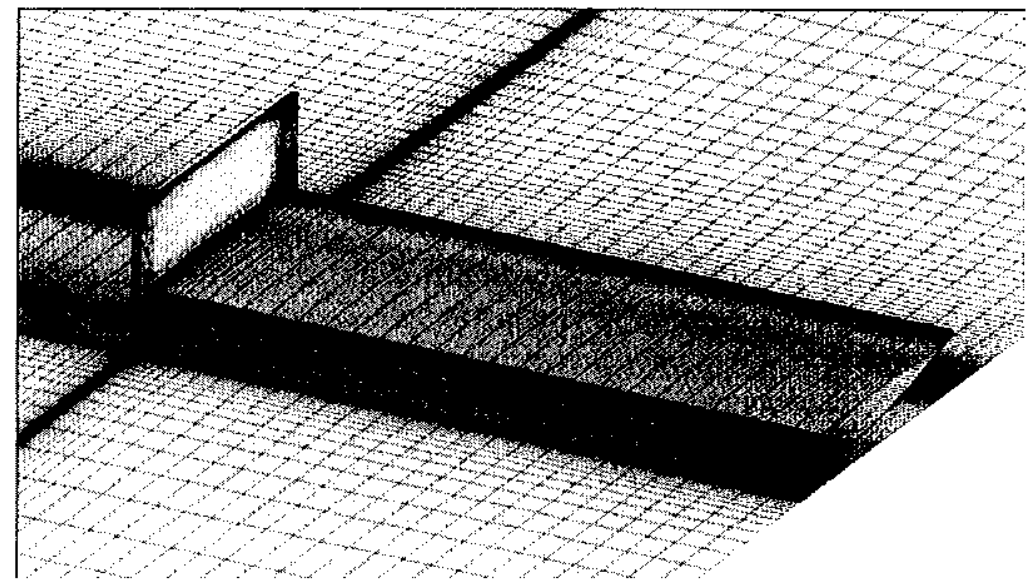

The coarse mesh featured $2 \times 10^{6}$ cells; 130 cells around the wing in the chordwise sense ( 3 across the blunt trailing edge) and 30 spanwise cells, spaced such that the endplate wall region was adequately resolved. The standard mesh, as shown in Figure 2, featured 190 cells around the wing ( 8 across the blunt trailing edge) and 45 spanwise cells, for a total of $3.11 \times 10^{6}$ volumes.

The finest mesh featured a greater cell density in the endplate and wake regions to investigate whether the endplate vortices were being sufficiently resolved (and thus influencing the lift and drag forces obtained); it featured 195 cells around the wing and 80 spanwise cells, with a finer spacing in the wake region to approximately $2 \mathrm{c}$ from the trailing edge. The total number of cells was $7.65 \times 10^{6}$. All the results were produced using the Realisable k- $\varepsilon$ turbulence model with enhanced wall modelling (Shih et al., 1995); this model has been shown in several recent studies on the same wing to be the most acceptable fully-turbulent model for reproducing experimental results (Mahon and Zhang, 2006; Doig et al., 2011; Diasinos et al., 2013), and therefore was deemed appropriate for the current investigation.

Table 2 Mesh refinement and force coefficients as compared to experimental results

\begin{tabular}{lcc}
\hline Mesh (no. volumes) & $C_{L}$ & $C_{D}$ \\
\hline $2.00 \times 10^{6}$ & 1.21 & 0.06 \\
$3.11 \times 10^{6}$ & 1.24 & 0.057 \\
$7.65 \times 10^{6}$ & 1.24 & 0.056 \\
(cxpcrimental) & 1.28 & 0.0545 \\
\hline
\end{tabular}

Source: Zerihan (2001) 
Predicted lift and drag coefficients, compared to the experimental results in Table 2, show that the fine and standard meshes were within very close agreement (approx. $1 \%$ or less), but the coarse mesh overpredicted drag and underpredicted lift by a more notable margin. The higher drag is likely to be a consequence of having fewer cells in the immediate wake and on the blunt trailing edge, leading to an artificially thick near-wake. The standard mesh was therefore used for all subsequent runs in an effort to limit unnecessary computational expense.

For further validation, results are presented in comparison to the experimental pressure distributions (Figure 3), and lift and drag coefficients (Figure 4) for ground clearances of $\mathrm{h} / \mathrm{c}=0.313,0.179$ and 0.067 . The CFD reproduces the force trends very well (including the lift loss at approx. $\mathrm{h} / \mathrm{c}=0.1$ ), somewhat over-predicting drag and under-predicting lift at higher clearances. It is worth noting that compressible simulations reproduced these results to within less than $1 \%$ difference in coefficients, indicating that the compressible solver could converge adequately at the lowest Mach number, but also that some minor density changes were present in the flow resulting in slight differences in the solution pressure distributions.

Figure 3 Pressure distributions from $\mathrm{CFD}$ at $\mathrm{h} / \mathrm{c}=0.313,0.179$ and 0.067 as compared to experimental results

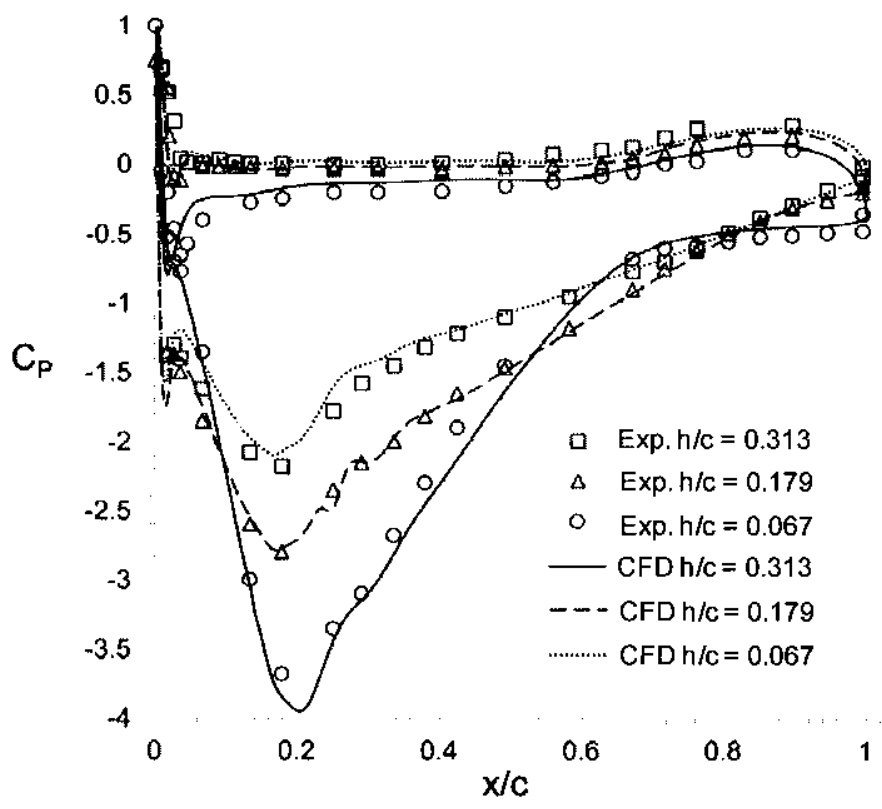

Source: Zerihan (2001) 
Figure 4 CFD predictions of lift and drag coefficients as compared to experimental results

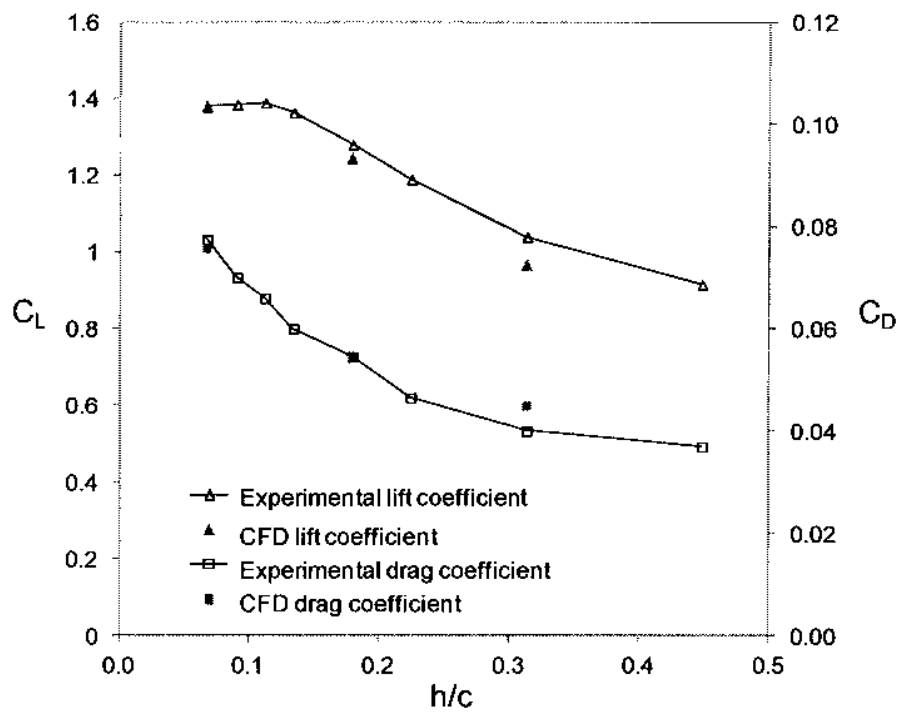

Source: Zerihan (2001)

The pressure coefficients obtained showed excellent agreement with the experimental results, with only a mild over-prediction of the suction peak at the lowest ground clearance of $\mathrm{h} / \mathrm{c}=0.067$ and under-prediction of the same point at $\mathrm{h} / \mathrm{c}=0.313$ (causing the under-prediction of lift at this clearance).

\section{Compressible simulations for Reynolds-scaled simulations}

Using the experiments of Zerihan (2001) as a starting point, this paper examines a scenario where the Reynolds number based on the wing chord is fixed at $0.459 \times 10^{6}$. This allows compressibility and Mach number effects to be divorced from the influence of Reynolds number.

While the Reynolds number may be exactly scaled, compressibility effects are non-linear and the difference in Mach number may skew the comparison, particularly when incompressible simulations are used (as is common practice currently in automotive fields). As computing power continues to increase and the expense of large clusters reduces, the time-saving represented by running incompressible flow simulations may be considered relatively small and compressible flow simulations may become a more viable prospect. However, it is more likely that the ability to test and examine a greater number of part variants would take precedence unless it was clear that the incompressible CFD was fundamentally unsuitable. 
The T026 wing used by Zerihan (2001), was tested at $\mathrm{h} / \mathrm{c}=0.313,0.179$ and 0.067 as in the validation cases discussed previously: clearances low enough to produce significantly increased peak local Mach numbers as compared to a freestream case, and representative of typical wing section ground clearances that might be examined in a wind tunnel test. As outlined in Table 1, the oncoming flow and ground speed of $30 \mathrm{~ms}^{-1}$ $\left(\mathrm{M}_{\infty}=0.0882\right)$ for the standard-size wing of Zerihan (2000) was Reynolds-scaled for $\operatorname{Re}=0.459 \times 10^{6}$ at $50 \%$ for a freestream of $60 \mathrm{~ms}^{-1}\left(\mathrm{M}_{\infty}=0.179\right)$, and then again at $25 \%$ scale for a freestream of $120 \mathrm{~ms}^{-1}\left(\mathrm{M}_{\infty}=0.352\right)$. The latter scale could be deemed as a more likely prospect for university-level research, where large wind tunnels with rolling roads are uncommon.

\subsection{Freestream comparison case}

In order to assess the extent to which ground proximity is influential in producing differences between incompressible and compressible CFD simulations, the three Reynolds-scaled cases were run for a 'freeflight' (no ground) case. Figure 5 shows that compressible $C_{D}$ does not change significantly from $M_{\infty}=0.0882$ to $M_{\infty}=0.1764$, and corresponds closely to the incompressible result (reducing by a fraction which would be within the bounds of numerical error between compressible and incompressible simulations). At $\mathrm{M}_{\infty}=0.3528$, the compressible $\mathrm{C}_{\mathrm{D}}$ is approximately $3.5 \%$ greater. The compressible $\mathrm{C}_{\mathrm{L}}$ in Figure 6 exhibits a small difference at $\mathrm{M}_{\infty}=0.0882$, increasing to around $1 \%$ at $M_{\infty}=0.1764$ and $3.7 \%$ at $M_{\infty}=0.3528$. The pressure distributions for each case in Figure 7 indicate that the difference in lift at the highest Mach number is due almost exclusively to the more prominent suction peak at approximately $\mathrm{x} / \mathrm{c}=0.18$, where the air has been accelerated to its maximum peak local Mach number.

Figure 5 Compressible and incompressible drag coefficients for the wing in free-flight (no ground) at different scales

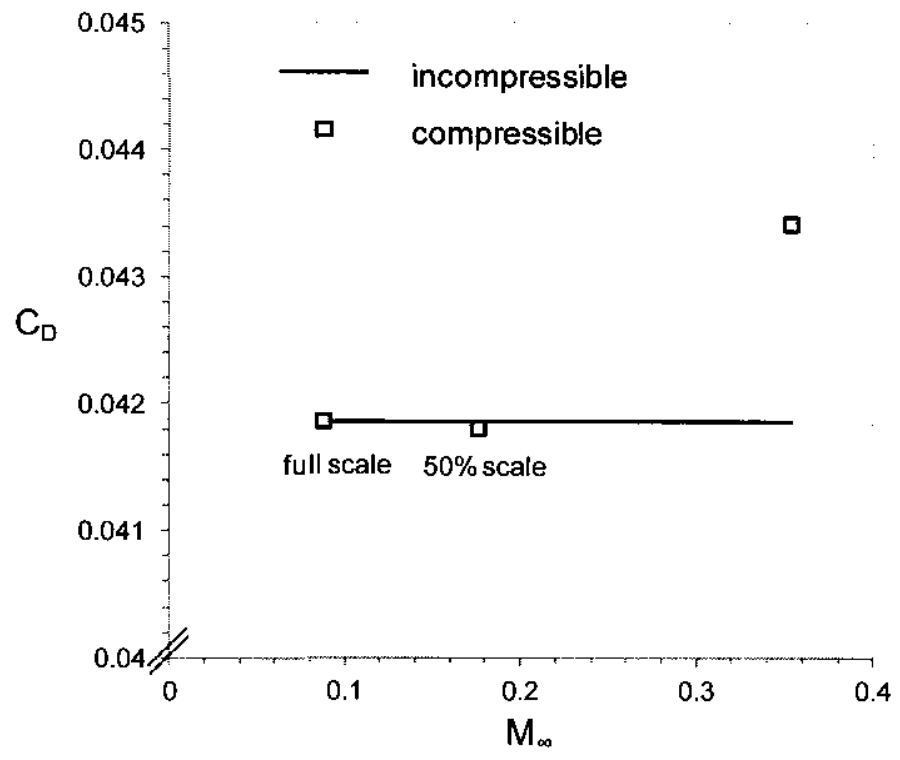


Figure 6 Compressible and incompressible lift coefficients for the wing in free-flight (no ground) at different scales

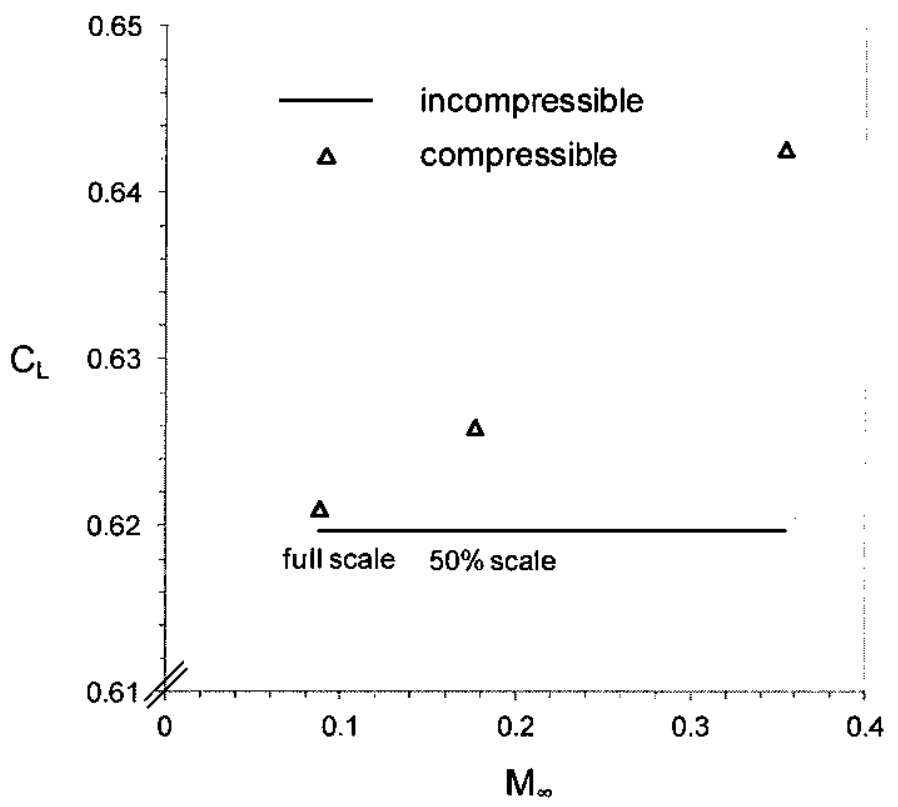

Figure 7 Compressible pressure coefficient distributions for the wing in free-flight (no ground) at scale, compared to the baseline full-scale incompressible result

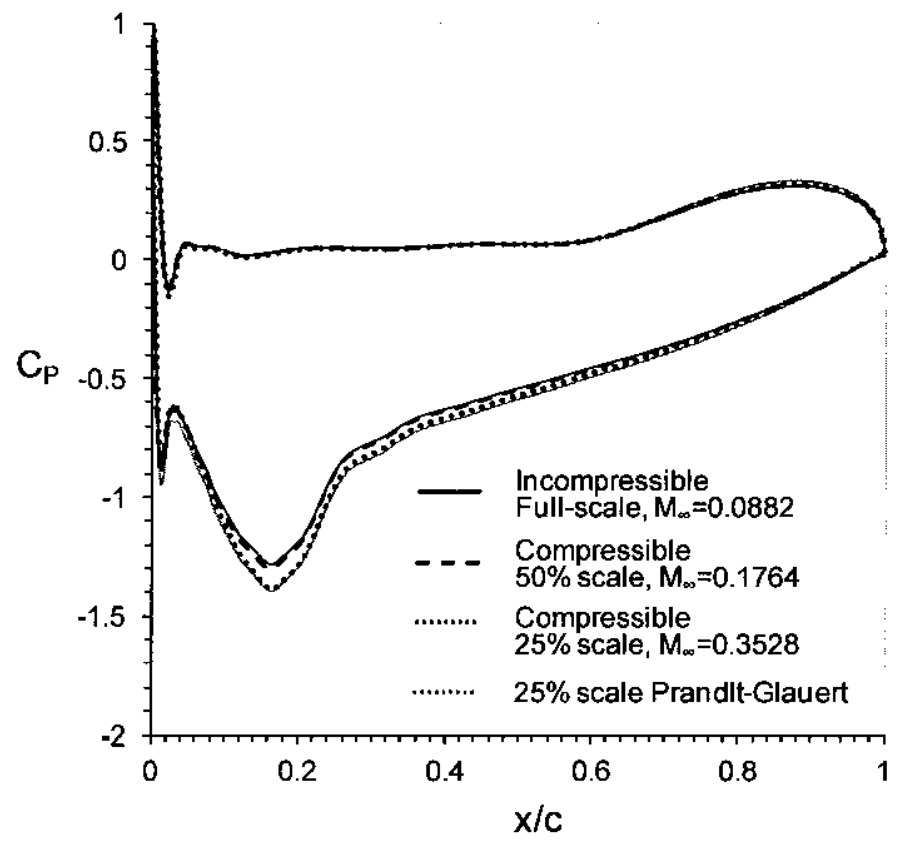


If we treat the compressible case as the most physically-realistic representation of the flowfield, resulting in the values of aerodynamic coefficients the wing would actually experience in the real world, it is clear that for a freestream Mach number of 0.3528 the incompressible assumption is no longer appropriate if good accuracy is to be achieved. This is consistent with the fact that the TO26 aerofoil section can be classified as high-lift, and therefore produces a considerable acceleration of the flow around its shape; indeed, the peak local Mach number for this compressible case is 0.547. Even before looking at the ground effect cases, it is obvious that the peak local Mach number in the presence of the ground will be considerably higher, and thus the trends observed in freestream will be exaggerated. The $25 \%$ scale model at $\mathrm{M}_{\infty}=0.3528$ represents a speed of $120 \mathrm{~ms}^{-1}$. This is slightly above that which almost all large-scale and advanced moving-ground tunnels can achieve, but it has been retained for comparison in the subsequent cases as the speed it represents is not far beyond the maximum of, for example, an Indycar Series racing car. It is certainly within the range of a vehicle such as a top-fuel dragster or land speed record car, which might be tunnel-tested at scale using, for instance, a less sophisticated elevated ground plane.

For comparison, a straightforward application of the Prandtl-Glauert rule is used on the incompressible result to account for the highest tested Mach number at $25 \%$ scale. Since the flow at the mid-span is relatively two-dimensional and the compressibility effects are mild, the correction proves to be excellent apart from the region where the leading edge curvature blends to the lower surface; therefore, in this instance, the Prandtl-Glauert rule could be applied with confidence for a simple correction.

\subsection{Ground effect cases}

In Figures 8 and 9, the influence of ground proximity on the lift and drag coefficients is revealed to be considerable, as it causes more significant differences in $C_{D}$ and $C_{L}$ respectively between incompressible and compressible cases than were obtained in the free-flight simulations.

At $\mathrm{M}_{\infty}=0.0882$, the drag predictions were negligibly different at all clearances, but the incompressible simulation under-predicted $C_{L}$ by around $0.5 \%$ for $h / c=0.179$ and over-predicts by the same amount for $\mathrm{h} / \mathrm{c}=0.067$. At $\mathrm{M}=0.1764$, the incompressible simulations under-predicted $C_{L}$ at the two higher clearances, and over-predicted at the lowest height. The non-linearity of compressibility effects is evident as the trends are extended to the $\mathrm{M}_{\infty}=0.3528$ cases, where lift is under-predicted by the incompressible simulations by $6.1 \%$, and drag by $13.9 \%$, at $\mathrm{h} / \mathrm{c}=0.179-\mathrm{a}$ broadly exponential increase in the margin by which the incompressible simulations are underestimating the force coefficients. For the lowest ground clearance, the marked increase in local Mach number at the suction peak leads to an increasingly strong pressure gradient that results in markedly enhanced separation towards the trailing edge - this results in the predicted lift being over-predicted by over $10 \%$ by the incompressible simulation, and drag being under-predicted by around $15 \%$. 
Figure 8 Compressible (C) and incompressible (I) drag coefficients for the wing at scale and for ground clearances of $\mathrm{h} /=0.313,0.179$ and 0.067

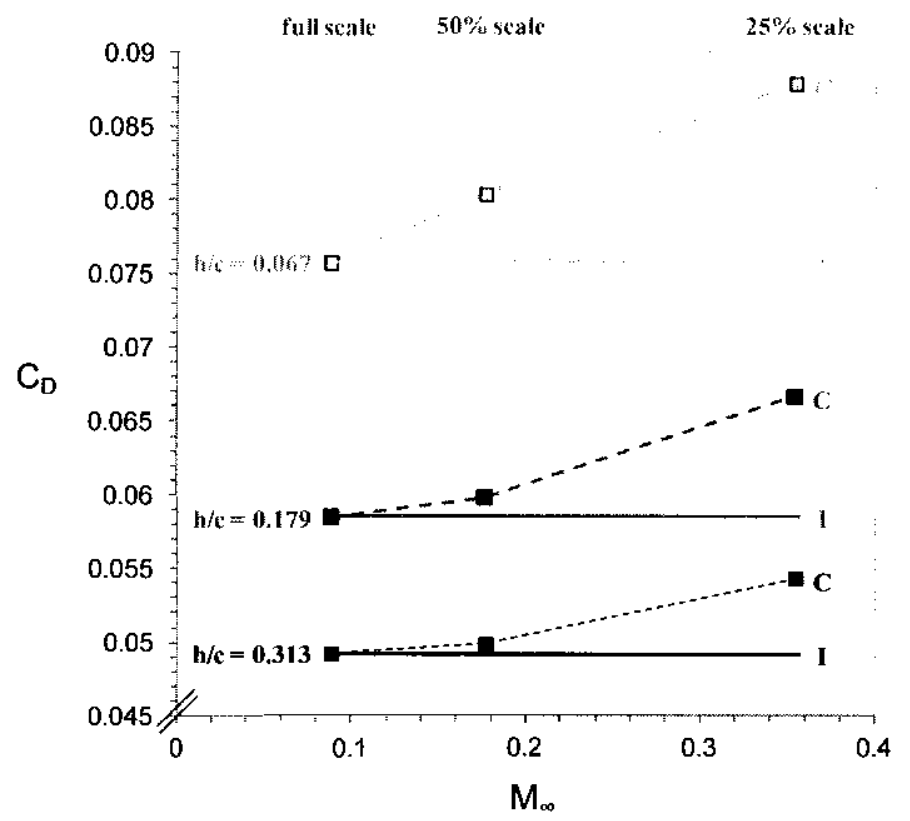

Figure 9 Compressible and incompressible lift coefficients for the wing at scale and for ground clearances of $\mathrm{h} /=0.313,0.179$ and 0.067

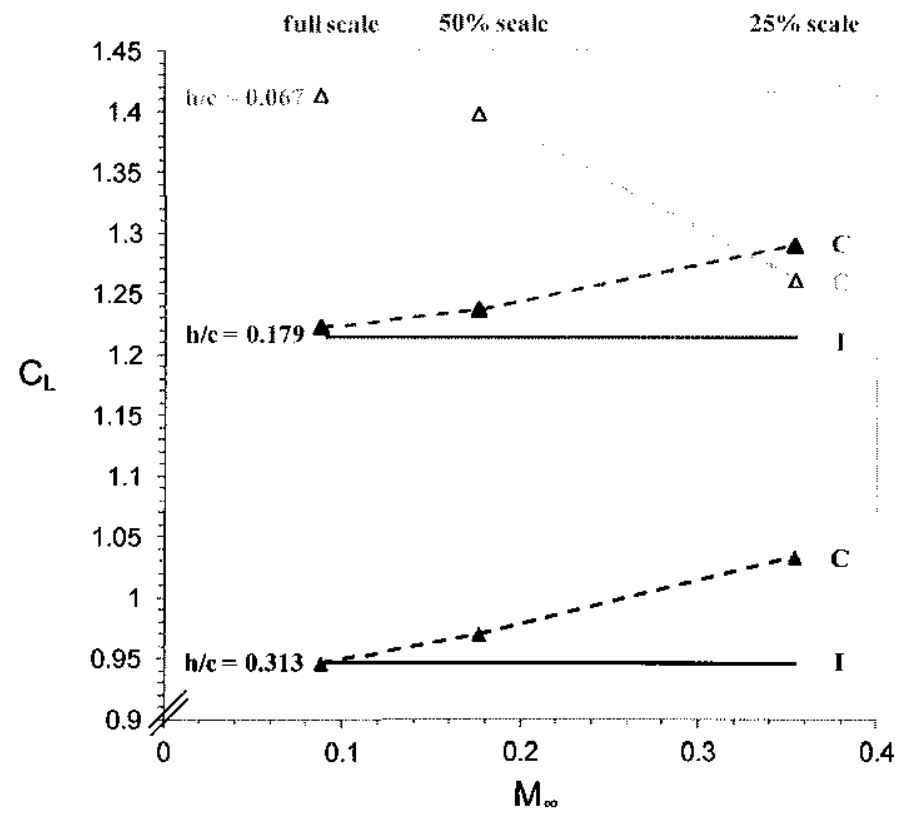


The results indicate that even at a relatively modest freestream Mach number of approximately 0.25 , one could be better served by Mach-scaling experiments to compare to incompressible CFD than by maintaining the Reynolds number regardless of the freestream velocity. In other words, allowing the Reynolds number to increase as a consequence of a Mach number increase while maintaining the original model scale is a more appropriate way to approach the issue of direct comparison, beyond a certain freestream Mach number, than to continue to decrease the model scale while maintaining the same Reynolds number as the full-scale original. This would be accompanied by consequences for transition locations and behaviour, which will not be discussed here but could distort the interpretation of results further. Thus, even before further investigation, it is already clear that the most appropriate way to model the flow, perhaps at $\mathrm{M}_{\infty}=0.0882$ and certainly at $\mathrm{M}_{\infty}=0.1764$, is to consider the air as compressible in all simulations.

\subsection{Resultant pressure coefficient distributions}

From this point, the $h / c=0.179$ case will be used as indicative of the flow response to compressibility. To examine in more detail the influence the Mach number and therefore flow compressibility has on the force coefficient results obtained, it is instructive to look to the sectional pressure distributions presented in Figure 10 for the semi-span (symmetry plane).

Figure 10 Compressible vs. incompressible semi-span pressure coefficient distributions at $\operatorname{Re}=0.459 \times 10^{6}$ for $50 \%$ and $25 \%$ scale, referenced to full-scale incompressible at $\mathrm{h} / \mathrm{c}=0.179$

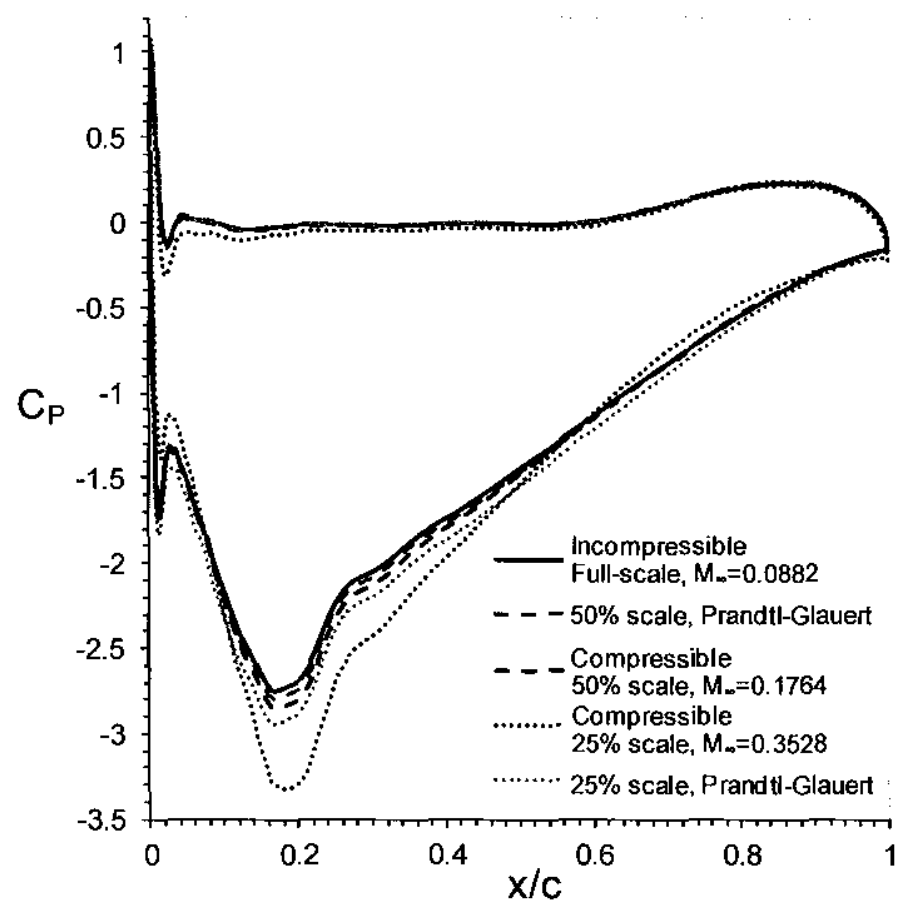


It is clear that the main cause for the differences noted between compressible and incompressible simulations stems from an incompressible under-prediction of the suction peak on the lower surface. This is present to a small degree at $M_{\infty}=0.1764$, extending across a longer chordwise stretch of the lower surface and altering the pressure gradient downstream to the trailing edge.

At the lower two Mach numbers, the upper-surface pressure distributions are similar. However, for the $25 \%$ scale model at $\mathrm{M}_{\infty}=0.3528$, the stagnation point has moved down such that the effective angle of oncoming flow just prior to the leading edge is decreased by almost $3^{\circ}$. From the full-scale to half-scale model, the stagnation point moves by less than $1 \%$ of the maximum section thickness, but from the full-scale to the quarter-scale geometry, the stagnation point moves downwards by a more significant $2.18 \%$ of maximum section thickness. This allows more flow over the upper surface, but is compensated in terms of negative lift by the additional acceleration between the wing and the ground such that this change to the oncoming flow angle that the wing experiences does not affect the wing's performance detrimentally overall. The lower-surface suction peak is significantly under-predicted by the incompressible simulation, and at the highest Mach number the extent of the rearwards adverse gradient is now considerably under-estimated as well, due to the changed trailing-edge conditions facilitated by the altered upper-surface $\mathrm{C}_{\mathrm{P}}$ distribution.

The primary driver for the differences is the ability of the density of the fluid to change as the Mach number is increased, and the extent of this is illustrated in Figure 11. Here, the density variations around the wing relative to the freestream value at the mid-span symmetry plane are clear, with close to a $6 \%$ variation in density at $M_{\infty}=0.1764$. While at this scale the peak local Mach number is less than $1 \%$ higher for the compressible simulation, it is enough to slightly alter the pressure gradient, whereas the $25 \%$ scale model experiences a significantly higher peak local Mach number (at a maximum of $\sim 0.79$ ). As the variations are highly three-dimensional in character, the structural response of the wing would also be affected, leading to different twist behaviour to that which would be expected from incompressible results.

This three-dimensionality, coupled with the increasing influence of separation at the trailing edge discussed below, means that the Prandtl-Glauert rule breaks down in its ability to apply a simple correction to the incompressible result, and Figure 10 highlights the underprediction of the suction peak by a considerable margin, as well as discrepancies close to the leading edge and towards the rear where the onset of separation has been exaggerated. Göthert's (1946) extension to the rule for three-dimensional flows would be a more appropriate treatment, but is similarly limited to slender bodies featuring attached flow. Therefore a full comparison of this more complex transformation has not been implemented here, particularly since a full vehicle features many bluff aspects and strongly vortex-influenced components that would be subject to compressible effects (Keogh et al., 2012).

As shown in Figure 12, the $M_{\infty}=0.3528$ (25\% scale) case experiences a notable separation near the trailing edge. The overlays on the lower surface density distributions on Figure 11 shows the regions in which the $x$-direction shear stress on the wing equals zero, indicating that the separation develops in the centre region of the wing, and remains attached at the endplate where Mach numbers are lower due to the vortices present there. These results indicate that any full-scale wing in ground effect which is designed for peak performance close to the point at which flow will separate could well experience 
significant flow separation in wind tunnel tests at a higher Mach number for the same Reynolds number being used for full-scale CFD. This creates the potential for considerable discrepancies when compared even to compressible full-scale simulations if the Mach number remains mismatched, and has implications for the flow reaching vehicle components which lie downstream of the wing. It is also conceivable that conflicting data about flow separation location would be obtained, and this is often a vital parameter for the effectiveness of a wing element and a leading reason for wind tunnel investigation.

Figure 11 Non-dimensional density variations (relative to freestream) around the wing on the symmetry plane (left) and on the lower surface (right) for the three Reynolds-scaled cases
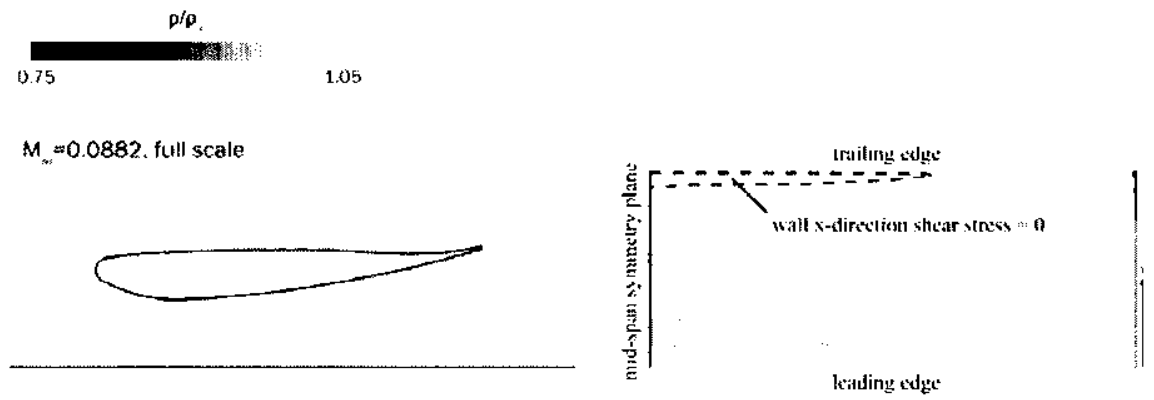

$M_{c}=0.1764,50 \%$ scale
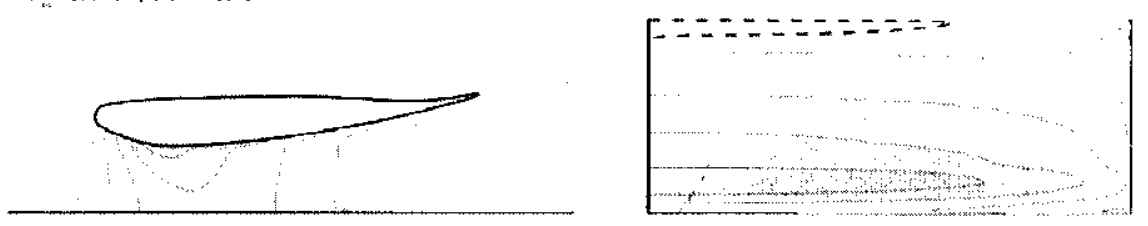

$M_{-}=0.3528,25 \%$ scale
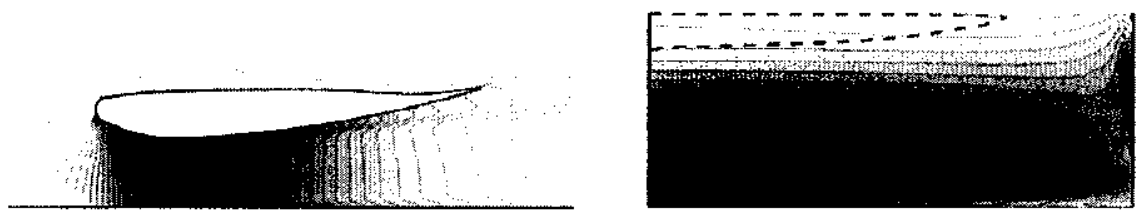

Figure 12 Streamlines around the trailing edge at the symmetry plane for (a) $50 \%$ scalc and (b) $25 \%$ scale compressible cases for $\mathrm{h} / \mathrm{c}=0.179$ (see online version for colours)

a)

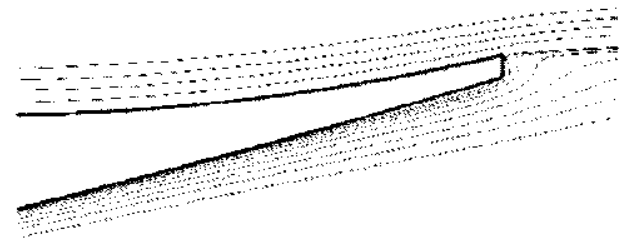

b)

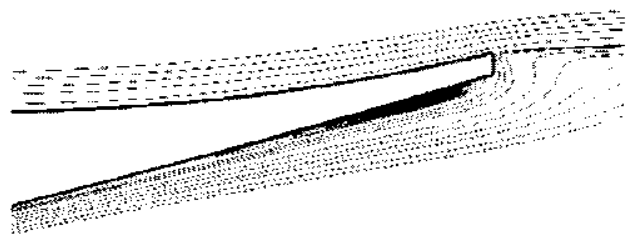


The peak local Mach numbers achieved by the flow between the wing and the ground, shown in Figure 13, are significantly higher than those for the free-flight case, where the peak accelerated flow only reached 1.55 times the freestream $\mathrm{M}_{\infty}=0.3528$ for the $25 \%$ scale case. The maximum $M_{\infty} / M$ for the full-scale model at $M_{\infty}=0.0882$ is equal to 1.79 , and at $\mathrm{M}_{\infty}=0.1794$ this value is 1.81 . This could not be considered a significant discrepancy in itself although in real terms the peak local Mach number in the $50 \%$ case is 0.33 and therefore already pushing into the compressible regime. At $25 \%$ scale, the flow accelerates to a local peak 2.12 times the freestream, and the actual peak Mach number is therefore 0.748 , indicating that the wing is not far from reaching its critical Mach number for this ground clearance and angle of attack.

Figure 13 Non-dimensional Mach number variations (relative to frecstream) around the wing on the symmetry plane for $50 \%$ and $25 \%$ scale compressible cases at $h / c=0.179$
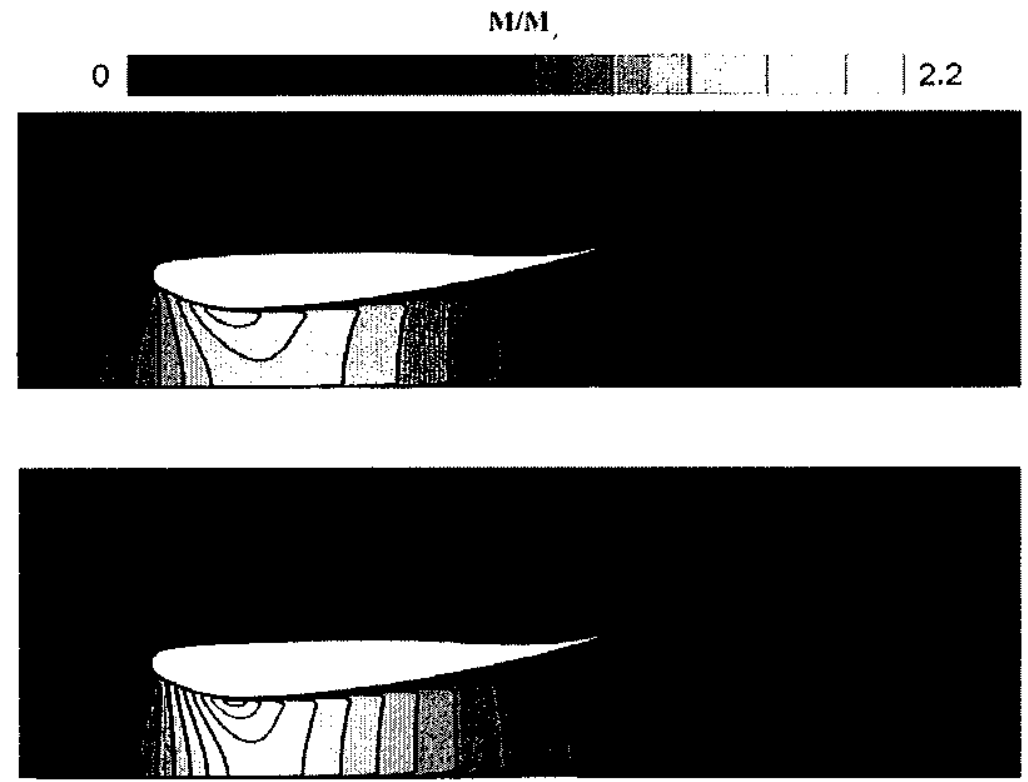

\subsection{Vortex and wake behaviour}

A comparison of the behaviour of the lower vortex from the wing/endplate junction is presented in Figures 14(a) and 14(b), with the vortex path assessed at planes 1c, 1.2c, $1.4 \mathrm{c}, 1.6 \mathrm{c}, 1.8 \mathrm{c}, 2 \mathrm{c}, 2.5 \mathrm{c}, 3 \mathrm{c}, 4 \mathrm{c}$ and $5 \mathrm{c}$ downstream of the trailing edge. The plot shows that the path of the vortex remains largely unaffected by the increase in freestream Mach number and decreasing scale. The figures show the $y$ and $z$ positions of the vortex changing by only small increments from case to case, though an exaggeration of the general trend is present for the $25 \%$ scale case at the highest Mach number. This remains a fairly minor difference until around two chord lengths downstream of the wing trailing edge, and on an actual open-wheel racing car the vortex would have, by this point, interacted in some way with the front wheels. 
Figure 14 Lower vortex core path moving downstream in (a) the $x-y$ plane and (b) the $x-z$ plane for $h / c=0.179$
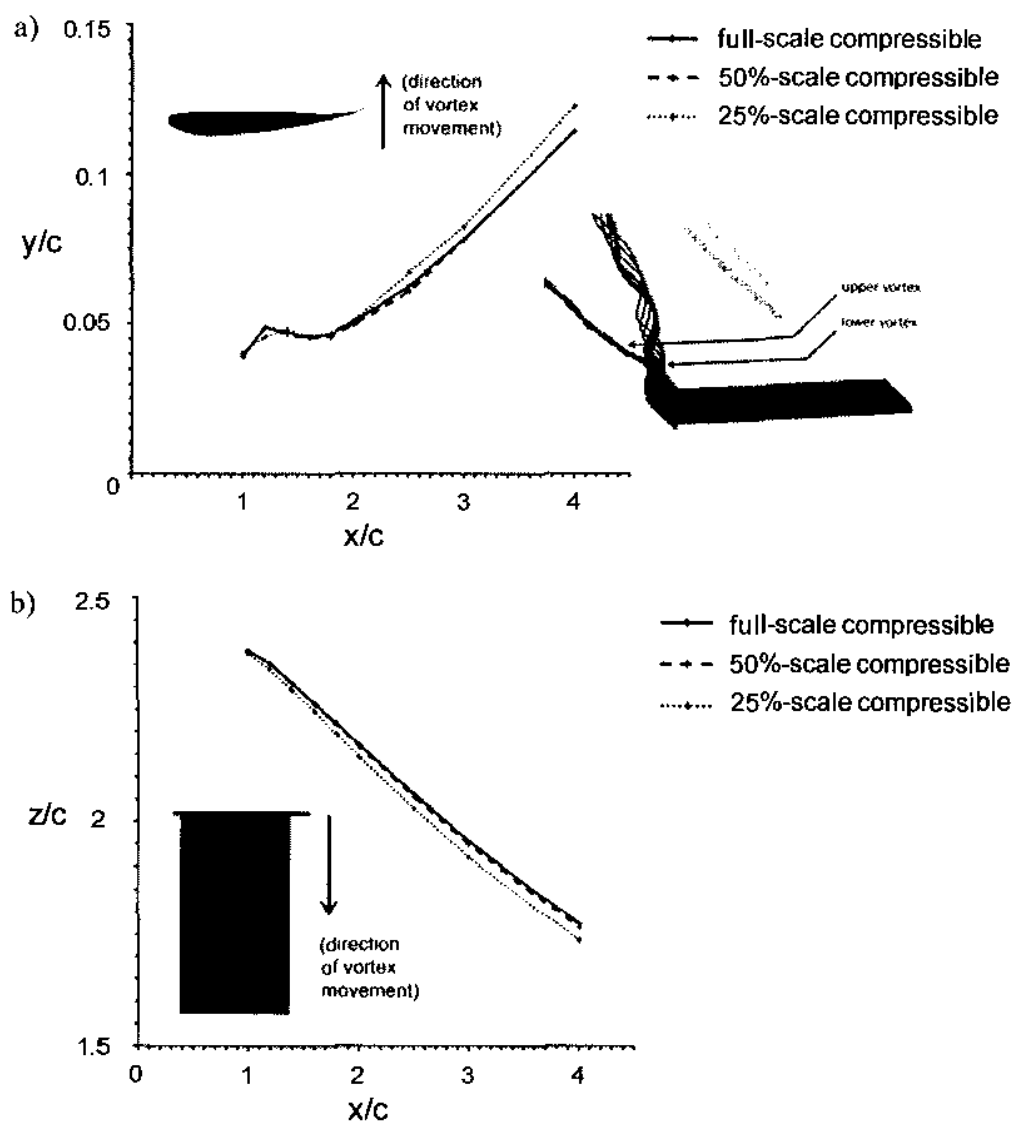

Figure 15 presents $\mathrm{u} / \mathrm{U}_{\infty}$ wake profiles for all the incompressible and compressible cases compared in this paper, at $1.2 \mathrm{c}$ and $2 \mathrm{c}$ from the leading edge, respectively (i.e. $0.2 \mathrm{c}$ and $1 \mathrm{c}$ downstream of the training edge). The profiles in both instances for all the incompressible cases are almost exactly identical, as one would expect from the other results discussed to this point. At full scale and $\mathrm{M}_{\infty}=0.0882$, the wake profiles for compressible and incompressible cases are essentially similar, with the compressible case predicting a slightly higher peak velocity deficit $(<0.1 \%$ greater $)$ at $1.2 \mathrm{c}$ and a more substantial deficit by $2 \mathrm{c}\left(0.9 \%\right.$ greater). Comparing the two $50 \%$ scale, $\mathrm{M}_{\infty}=0.1764$ cases, the velocity deficit differences have increased to $2.5 \%$ at $1.2 \mathrm{c}$ and $1.2 \%$ at $2 \mathrm{c}$. The compressible centreline of the wake is also situated subtly lower than its incompressible counterpart, as a result of the mild increase in pressure difference caused by the additional acceleration of the compressible flow under the wing. 
Figure 15 Streamwise velocity wake profiles on the symmetry plane at (a) $1.2 \mathrm{c}$ and (b) $2 \mathrm{c}$ from the leading edge for $h / c=0.179$

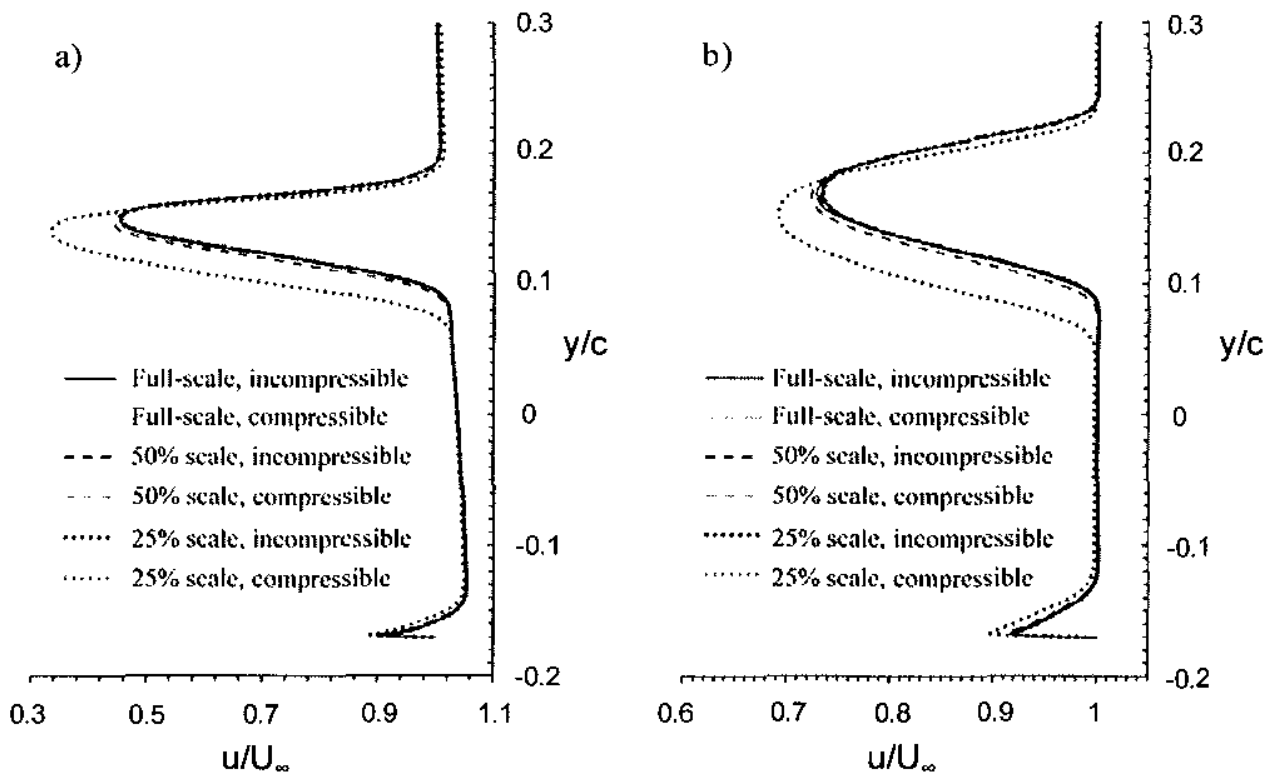

This effect is magnified when the oncoming Mach number is increased to 0.3528 and the scale reduced to $25 \%$. The wake is visibly and distinctly stronger, thicker, and closer to the ground, consistent with the enhanced separation observed previously. This confirms that the primary cause of the marked increase in predicted drag is the effect on the wake of the increased pressure gradient of the lower surface.

Given the minor differences in vortex behaviour but the more substantial incongruities in the streamwise wake profiles behind the wing on the symmetry plane, the flow reaching any components downstream of the wing (i.e., the wheels, suspension or sidepods of an open-wheel race car) would experience conditions for higher freestream Mach numbers which do not conform closely to those seen at the lowest freestream Mach number.

\subsection{Further comments}

Although it is possible that the Reynolds and Mach number scaling effects establish themselves in different fashions in the flow, leading to a misleading constructive or destructive interference between the two, Mach scaling in most large-scale subsonic tunnels would involve impractical or prohibitively-expensive additional equipment to modify the pressure and temperature being delivered to the test section. For this reason this possibility has not been pursued further here. One can conclude from the data presented that the only way to achieve truly effective validation between experiments and CFD for inverted wings even at relatively low Mach numbers is to ensure the computational model matches the experiment as closely as possible; that is, not only should the Reynolds number be matched, but the scale and Mach number should be comparable and, given that tunnel blockage would exaggerate any compressible effects further, the test section geometry should be represented with reasonable fidelity in the 
computational model as well. Accepting the considerable influence which compressibility has on the flow even at the lowest end of the range of freestream velocities examined here, unlike for the same wing in free (unbounded) flight or a section which is much less cambered than the TO26, one can see that running all CFD as compressible is desirable in order to obtain the most accurate simulation results with which to compare to experimental results.

It also appears likely that other racing car components, such as wheels, rear wings, diffusers, etc. will atso experience significant compressibility, and the cumulative effect of many small discrepancies, when comparing tunnel tests or track data to incompressible $\mathrm{CFD}$, may culminate in differences in result sets which should not be ignored.

\section{Conclusions}

Compressible effects can affect the results of Reynolds-scaled wind tunnel tests on models which experience a higher freestream Mach number in order to maintain a Reynolds number consistent with a full scale model in CFD or an actual vehicle on track. In this study, the Reynolds number of flow around an inverted wing was maintained at $0.459 \times 10^{6}$ as the model was scaled at $50 \%$ and $25 \%$, the former of which is very common in race car wind tunnel testing.

The discrepancy at $50 \%$ scale and a Mach number of 0.1764 , compared to a full-scale model at Mach 0.0882 , was $1 \%$ and $3.7 \%$ in terms of drag and lift respectively, and the differences increased non-linearly as the Mach number was increased again for a $25 \%$ scale model. As the incompressible simulation is essentially similar to the compressible simulation at the lowest Mach number, treating the flow as incompressible underestimates the aerodynamic coefficients by the same amount as failing to Mach-scale the model. A simple Prandtl-Glauert compressibility correction fails to predict the magnitude of discrepancies in ground effect for centre-span pressure coefficients, and a three-dimensional equivalent would be difficult to apply to a full vehicle with confidence; this underscores the need to perform full compressible simulations in order to obtain more accurate results.

Compressible effects on, in particular, transition and separation, would be better examined using large-eddy simulation. While increasing the computational cost of simulation beyond that which even the leading teams can currently contemplate, in the future this will continue to become more feasible and from a pure research point of view is achievable and pertinent for subsequent studies.

\section{References}

Diasinos, S., Barber, T.J. and Doig, G. (2013) 'Influence of wing span on the aerodynamics of wings in ground effect', Proceedings of the Institution of Mechanical Engineers, Part G: Journal of Aerospace Engineering, Vol. 227, No. 3, pp.569-573.

Doig, G., Barber, T.J. and Neely, A.J. (2011) 'The influence of compressibility on the aerodynamics of an inverted wing in ground effect', Journal of Fluids Engineering, Vol. 133, No. 6, pp.061102-01-061102-12.

Doig, G., Barber, T.J., Leonardi, E. and Neely, A.J. (2007) 'The onset of compressibility effects for an inverted aerofoil in ground effect', The Aeronautical Journal, Vol. 111, No. 1126, pp.797-806. 
FIA (2013) Formula I Sporting Regulations, Section 22, Fédération Internationale de l'Automobile [online] http://www.formulal.com/inside_fl/rules_and_regulations/sporting_regulations/ 8713/.

Glauert, H. (1928) 'The effect of compressibility on the lift of an aerofoil', Proc. Roy. Soc., London, Vol. 118, No. 779, pp.113-119.

Göthert, B.H. (1946) Plane and Three-Dimensional Flow at High Subsonic Speeds, Extension of the Prandtl Rule, NACA TM 1105.

Keogh, J., Doig, G. and Diasinos, S. (2012) 'The influence of compressibility effects in correlation issues for aerodynamic development of racing cars', 18th Australiasian Fluid Mechanics Conference, Launceston, 3-7 December.

Mahon, S. and Zhang, X. (2006) 'Computational analysis of pressure and wake characteristics on an aerofoil in ground effect', ASME Journal of Fluids Engineering, Vol. 127, No. 2, pp. $290-298$.

Ranzenbach, R. (1995) 'Cambered airfoil in ground effect-wind tunnel and road conditions', Proceedings of the 13th AIAA Applied Aerodynamics Conference, San Diego, USA, pp. 1208-1215.

Ranzenbach, R. and Barlow, J. (1994) Two Dimensional Aerofoil in Ground Effect, Experimental and Computational Study, SAE Paper 942509.

Ranzenbach, R. and Barlow, J. (1995) Cambered Airfoil in Ground Effect-Wind Tunnel and Road Conditions, AIA^ Paper 95-1909-CP.

Ranzenbach, R. and Barlow, J. (1996) Cambered Airofoil in Ground Effect - An Experimental and Computational Study, S^E Paper 960909.

Ranzenbach, R. and Barlow, J. (1997) Multielement Aerofoil in Ground Effect, Experimental and Computational Study, AIAA Paper 97-2238

Shih, T.H., Liou, W.W., Shabbir, A., Yang, Z. and Zhu, J. (1995) 'A new k- $\varepsilon$ eddy-viscosity model for high Reynolds number turbulent flows - model development and validation', Computers $\&$ Fluids, Vol. 24, No. 3, pp.227-238.

Sutherland, W. (1893) 'The viscosity of gases and molecular force', Philosophical Magazine, Vol. 5, No. 36, pp.507-531.

Toet, W. (2013) 'Aerodynamics and aerodynamic research in Formula 1', The Aeronautical Journal, Vol. 117, No. 1187, pp.1-26.

Zerihan, J. (2001) An Investigation into the Aerodynamics of Wings in Ground Effect, PhD Thesis, University of Southampton, School of Engineering Sciences, UK.

Zhang, X., Toct, W. and Zerihan, J. (2006) 'Ground effect aerodynamics of race cars', Applied Mechanics Reviews, Vol. 59, No. 1, pp.33-49. 